\title{
Higgsino and gaugino pair production at the LHC with aNNLO + NNLL precision
}

\author{
Juri Fiaschi $\odot^{*}$ and Michael Klasen $\circledast^{\dagger}$ \\ Institut für Theoretische Physik, Westfälische Wilhelms-Universität Münster, \\ Wilhelm-Klemm-Straße 9, 48149 Münster, Germany
}

(Received 4 June 2020; accepted 13 October 2020; published 20 November 2020)

\begin{abstract}
We present a calculation of Higgsino and gaugino pair production at the LHC at next-to-next-to-leading logarithmic (NNLL) accuracy, matched to approximate next-to-next-to-leading order (aNNLO) QCD corrections. We briefly review the formalism for the resummation of large threshold logarithms and highlight the analytical results required at aNNLO + NNLO accuracy. Our numerical results are found to depend on the mass and nature of the produced charginos and neutralinos. The differential and total cross sections for light Higgsinos, which like sleptons are produced mostly at small $x$ and in the $s$-channel, are found to be again moderately increased with respect to our previous results. The differential and total cross sections for gauginos are, however, not increased any more due to the fact that gauginos, like squarks, are now constrained by ATLAS and CMS to be heavier than about $1 \mathrm{TeV}$, so that also $t$ - and $u$-channels play an important role. The valence quarks probed at large $x$ then also induce substantially different cross sections for positively and negatively charged gauginos. The Higgsino and gaugino cross sections are both further stabilized at aNNLO + NNLL with respect to the variation of renormalization and factorization scales. We also now take mixing in the squark sector into account and study the dependence of the total cross sections on the squark and gluino masses as well as the trilinear coupling controlling the mixing in particular in the sbottom sector.
\end{abstract}

DOI: 10.1103/PhysRevD.102.095021

\section{INTRODUCTION}

The minimal supersymmetric (SUSY) Standard Model (MSSM) is a theoretically and phenomenologically well motivated extension of the Standard Model (SM) of particle physics, that can solve a significant number of shortcomings of this model [1,2]. Important examples in this respect are the stabilization of the Higgs boson mass and the unification of strong and electroweak forces at high scales. The MSSM predicts fermionic partners of the neutral and charged gauge and Higgs bosons called gauginos and Higgsinos, which are typically among the lightest SUSY particles [3]. The lightest neutral mass eigenstate, the lightest neutralino, is one of the best studied dark matter candidates [4-12]. Heavier neutralinos and charginos decay typically into multilepton final states and missing transverse momentum. Searches for Higgsino[13-18] or gaugino-like particles [19-24] are therefore

\footnotetext{
fiaschi@uni-muenster.de

†michael.klasen@uni-muenster.de
}

Published by the American Physical Society under the terms of the Creative Commons Attribution 4.0 International license. Further distribution of this work must maintain attribution to the author(s) and the published article's title, journal citation, and DOI. Funded by SCOAP . important physics goals at the LHC. They are often carried out in the framework of simplified models $[25,26]$. Care must, however, be taken that the theoretical assumptions are not overly simplified [27].

Experimental measurements of supersymmetric (SUSY) production cross sections at past and future runs of the LHC require precise theoretical calculations at the level of nextto-leading order (NLO) QCD and beyond [28-37]. In the perturbative expansion, logarithmically enhanced terms appear beyond leading order in the strong coupling constant $\alpha_{s}$, whose contributions can be sizeable close to production threshold or at small transverse momentum of the produced SUSY particle pair. Their effect on neutralino, chargino [38-43], slepton [44-49], squark, gluino [50-53], stop [54,55] and also new gauge boson production [56-59] has been taken into account to all orders with resummation techniques to next-to-leading logarithmic (NLL) accuracy and beyond. The results for the electroweak production channels have been made publicly available with the code RESUMMINO [60] and are regularly employed in the experimental analyses by ATLAS [23] and CMS [13]. Predictions have also recently been made for the highluminosity (HL) and high-energy (HE) phases of the LHC [61]. The effect of higher order QCD corrections is generally to enhance the theoretical estimations for the cross sections, while on the other hand they reduce the 
dependence of the results on the choice of the unphysical renormalization and factorization scales. Together with resummation-improved parton density functions (PDFs) [62], also the PDF uncertainty can in principle be reduced [63-67], even though in practice these PDFs must currently be fitted to smaller data sets than global NLO analyses and thus still have larger errors.

In this paper, we take our precision calculations for Higgsino and gaugino pair production to the next level by resumming not only the leading and next-to-leading logarithms (NLL), but also the next-to-next-to-leading logarithms (NNLL) and matching them not only to the full NLO QCD and SUSY-QCD corrections, but also an approximate next-to-next-to-leading order (aNNLO) calculation in QCD. The corresponding analytical formulas are available in the literature [68-71], so that we collect here only the most important results required at NNLL accuracy. Similar calculations, based on full NLO SUSY-QCD and aNNLO QCD calculations [28,29], have also been performed previously for sleptons [71] as well as for squarks, gluinos [50] and stops [55] and are available through the public codes RESUMMINO [60] and NNLL-fast [53]. Other groups have employed soft-collinear effective theory for sleptons [45], squarks [72], gluinos [52], and stops [54,73] with similar conclusions.

The paper is organized as follows: In Sec. II, we present our analytical approach and in particular how threshold logarithms can be resummed at NNLL accuracy, matched to a fixed-order calculation up to NNLO and how the PDFs and hadronic cross sections are transformed to and from Mellin space. Our numerical results for the production of relatively light Higgsino pairs are contained in Sec. III. This section starts with a discussion of the QCD and SUSY input parameters, followed by a demonstration of how the NNLL and aNNLO contributions affect the differential cross section at small and large invariant masses. We then show the effects of the new contributions on the total cross section and its dependence on the factorization and renormalization scales. We also discuss the dependence on other SUSY parameters like the squark and gluino masses and the trilinear coupling governing squark mixing in the bottom sector. Numerical results for the pair production of heavier gauginos are described in a similar way in Sec. IV. The ensuing conclusions are presented in Sec. V.

\section{ANALYTICAL APPROACH}

The hadronic invariant mass distribution for the pair production of neutralinos and charginos

$$
\begin{aligned}
M^{2} \frac{d \sigma_{A B}}{d M^{2}}(\tau)= & \sum_{a, b} \int_{0}^{1} d x_{a} d x_{b} d z \\
& \times\left[x_{a} f_{a / A}\left(x_{a}, \mu_{F}^{2}\right)\right]\left[x_{b} f_{b / B}\left(x_{b}, \mu_{F}^{2}\right)\right] \\
& \times\left[z \sigma_{a b}\left(z, M^{2}, \mu_{R}^{2}, \mu_{F}^{2}\right)\right] \delta\left(\tau-x_{a} x_{b} z\right)
\end{aligned}
$$

requires the convolution of parton density functions (PDFs) $f_{a, b / A, B}$ with the partonic cross section $\sigma_{a b}$. The former depend on $x_{a, b}$, the longitudinal momentum fractions of the partons $a$ and $b$ in the colliding hadrons $A$ and $B$, and the factorization scale $\mu_{F}$. The latter is a function of the squared invariant mass of the produced neutralinos or charginos $M^{2}$, its ratio $z=M^{2} / s$ to the partonic center-of-mass energy $s$, and the renormalization and factorization scales $\mu_{R}$ and $\mu_{F}$. In contrast to the leading order (LO) cross section $[74,75]$ and the virtual next-to-leading order (NLO) corrections, which are proportional to $\delta(1-z)$ [30], the kinematic mismatch in the cancellation of infrared divergences among the virtual and real corrections of order $n$ introduces large logarithmic remainders proportional to

$$
\alpha_{s}^{n}\left(\mu_{R}^{2}\right)\left[\frac{\ln ^{m}(1-z)}{1-z}\right]_{+}, \quad \text { where } m \leq 2 n-1 .
$$

Close to threshold $(z \rightarrow 1)$, they spoil the convergence of the perturbative series in $\alpha_{s}$ and therefore have to be resummed to all orders [76,77].

After performing a Mellin transformation of the PDFs and partonic cross section in Eq. (1), the hadronic cross section $\sigma_{A B}$ factorizes, the singular terms in Eq. (2) turn into large logarithms of the Mellin variable $N$,

$$
\left[\frac{\ln ^{m}(1-z)}{1-z}\right]_{+} \rightarrow \ln ^{m+1} N+\ldots,
$$

and the partonic cross section $\sigma_{a b}$ can be written in the exponentiated form

$$
\begin{aligned}
\sigma_{a b}^{(\text {res })}\left(N, M^{2}, \mu_{R}^{2}, \mu_{F}^{2}\right)= & H_{a b}\left(M^{2}, \mu_{R}^{2}, \mu_{F}^{2}\right) \\
& \times \exp \left[G_{a b}\left(N, M^{2}, \mu_{R}^{2}, \mu_{F}^{2}\right)\right] \\
& +\mathcal{O}\left(\frac{1}{N}\right) .
\end{aligned}
$$

Here, the exponent $G_{a b}$ is universal and contains all the logarithmically enhanced contributions in the Mellin variable $N$, while the hard function $H_{a b}$ is independent of $N$, though process-dependent.

Up to next-to-next-to-leading logarithmic (NNLL) accuracy, the exponent $G_{a b}$ can be written as

$$
\begin{aligned}
G_{a b}\left(N, M^{2}, \mu_{R}^{2}, \mu_{F}^{2}\right)= & L G_{a b}^{(1)}(\lambda)+G_{a b}^{(2)}\left(\lambda, M^{2}, \mu_{R}^{2}, \mu_{F}^{2}\right) \\
& +\alpha_{s} G_{a b}^{(3)}\left(\lambda, M^{2}, \mu_{R}^{2}, \mu_{F}^{2}\right),
\end{aligned}
$$

where $\lambda=\alpha_{s} b_{0} L$ and $L=\ln \bar{N}=\ln \left(N e^{\gamma_{E}}\right)$. For DrellYan-like processes such as slepton or Higgsino and gaugino pair production initiated by quarks and antiquarks only, the coefficients $G_{a b}^{(i)}=g_{a}^{(i)}+g_{b}^{(i)}$ with $a=b=q$ can be found up to next-to-leading logarithmic (NLL) accuracy in Refs. $[40,47]$. In addition to the LL and NLL terms $g_{q}^{(1)}$ and $g_{q}^{(2)}$, one needs at NNLL also [68] 


$$
\begin{aligned}
g_{q}^{(3)}(\lambda)= & \frac{A^{(1)} b_{1}^{2}}{2 \pi b_{0}^{4}} \frac{1}{1-2 \lambda}\left[2 \lambda^{2}+2 \lambda \ln (1-2 \lambda)+\frac{1}{2} \ln ^{2}(1-2 \lambda)\right] \\
& +\frac{A^{(1)} b_{2}}{2 \pi b_{0}^{3}}\left[2 \lambda+\ln (1-2 \lambda)+\frac{2 \lambda^{2}}{1-2 \lambda}\right]+\frac{2 A^{(1)}}{\pi} \zeta_{2} \frac{\lambda}{1-2 \lambda} \\
& -\frac{A^{(2)} b_{1}}{(2 \pi)^{2} b_{0}^{3}} \frac{1}{1-2 \lambda}\left[2 \lambda^{2}+2 \lambda+\ln (1-2 \lambda)\right]+\frac{A^{(3)}}{\pi^{3} b_{0}^{2}} \frac{\lambda^{2}}{1-2 \lambda}-\frac{D^{(2)}}{2 \pi^{2} b_{0}} \frac{\lambda}{1-2 \lambda} \\
& +\frac{A^{(1)} b_{1}}{2 \pi b_{0}^{2}} \frac{1}{1-2 \lambda}[2 \lambda+\ln (1-2 \lambda)] \ln \left(\frac{M^{2}}{\mu_{R}^{2}}\right)+\frac{A^{(1)}}{2 \pi}\left[\frac{\lambda}{1-2 \lambda} \ln ^{2}\left(\frac{M^{2}}{\mu_{R}^{2}}\right)-\lambda \ln ^{2}\left(\frac{\mu_{F}^{2}}{\mu_{R}^{2}}\right)\right] \\
& -\frac{A^{(2)}}{2 \pi^{2} b_{0}}\left[\frac{\lambda}{1-2 \lambda} \ln \left(\frac{M^{2}}{\mu_{R}^{2}}\right)-\lambda \ln \left(\frac{\mu_{F}^{2}}{\mu_{R}^{2}}\right)\right] .
\end{aligned}
$$

Here, the new coefficients required at NNLL are given by [78]

$$
A^{(3)}=\frac{1}{2} C_{F}\left[C_{A}^{2}\left(\frac{245}{24}-\frac{67}{9} \zeta_{2}+\frac{11}{6} \zeta_{3}+\frac{11}{5} \zeta_{2}^{2}\right)+C_{F} n_{f}\left(2 \zeta_{3}-\frac{55}{24}\right)+C_{A} n_{f}\left(\frac{10}{9} \zeta_{2}-\frac{7}{3} \zeta_{3}-\frac{209}{108}\right)-\frac{n_{f}^{2}}{27}\right]
$$

and [68]

$$
D^{(2)}=2 C_{F}\left[C_{A}\left(-\frac{101}{27}+\frac{11}{3} \zeta_{2}+\frac{7}{2} \zeta_{3}\right)+n_{f}\left(\frac{14}{27}-\frac{2}{3} \zeta_{2}\right)\right]
$$

The coefficients of the QCD $\beta$-function are denoted by $b_{n}=\beta_{n} /(2 \pi)^{n+1} \quad[79,80]$, the QCD color factors are $C_{A}=N_{C}=3$ and $C_{F}=\left(N^{2}-1\right) /\left(2 N_{C}\right)=$ $4 / 3$, and the number of active quark flavors is $n_{f}=5$.

The hard $N$-independent part of the Mellin-transformed partonic cross section in Eq. (4)

$$
H_{a b}\left(M^{2}, \mu_{R}^{2}, \mu_{F}^{2}\right)=\sigma_{a b}^{(0)} \mathcal{C}_{a b}\left(M^{2}, \mu_{R}^{2}, \mu_{F}^{2}\right)
$$

can be perturbatively expanded in terms of the Mellintransformed LO cross section $\sigma_{a b}^{(0)}$ and

$$
\mathcal{C}_{a b}\left(M^{2}, \mu_{R}^{2}, \mu_{F}^{2}\right)=\sum_{n=0}\left(\frac{\alpha_{s}}{2 \pi}\right)^{n} \mathcal{C}_{a b}^{(n)}\left(M^{2}, \mu_{R}^{2}, \mu_{F}^{2}\right)
$$

where the hard matching coefficients

$$
\mathcal{C}_{a b}^{(n)}\left(M^{2}, \mu_{R}^{2}, \mu_{F}^{2}\right)=\left(\frac{2 \pi}{\alpha_{s}}\right)^{n}\left[\frac{\sigma_{a b}^{(n)}}{\sigma_{a b}^{(0)}}\right]_{\mathrm{N}-\text { ind }}
$$

are obtained from the finite ( $N$-independent) terms in the ratio of the $n$th order cross section over the LO one. The QCD part of the coefficient required at next-to-next-toleading order (NNLO) in pair-invariant mass kinematics is given by $[69,70]$

$$
\begin{aligned}
\mathcal{C}_{q \bar{q}}^{(2)}= & \frac{C_{F}}{720}\left\{5\left(-4605 C_{A}+4599 C_{F}+762 n_{f}\right)+20 \pi^{2}\left(188 C_{A}-297 C_{F}-32 n_{f}\right)\right. \\
& -92 \pi^{4}\left(C_{A}-6 C_{F}\right)+180\left(11 C_{A}+18 C_{F}-2 n_{f}\right) \log ^{2}\left(\frac{\mu_{F}^{2}}{M^{2}}\right) \\
& -160\left(11 C_{A}-2 n_{f}\right)\left(6-\pi^{2}\right) \log \left(\frac{\mu_{R}^{2}}{M^{2}}\right)+80\left(151 C_{A}-135 C_{F}+2 n_{f}\right) \zeta_{3} \\
& +20 \log \left(\frac{\mu_{F}^{2}}{M^{2}}\right)\left[-51 C_{A}+837 C_{F}+6 n_{f}-4 \pi^{2}\left(11 C_{A}+27 C_{F}-2 n_{f}\right)\right. \\
& \left.\left.+\left(-198 C_{A}+36 n_{f}\right) \log \left(\frac{\mu_{R}^{2}}{M^{2}}\right)+216\left(C_{A}-2 C_{F}\right) \zeta_{3}\right]\right\} .
\end{aligned}
$$


It allows to improve the resummation of logarithmically enhanced contributions, since also beyond NNLO in $\alpha_{s}$ the finite terms are multiplied by threshold logarithms.

While in the limit of large $N$ the cross section is clearly dominated by terms of $\mathcal{O}\left(\ln ^{2} N\right), \mathcal{O}(\ln N)$ and $\mathcal{O}(1)$, some of the terms suppressed by powers of $1 / N$ are multiplied by powers of $\ln N$ and can thus also have a non-negligible effect [81-87]. This collinear improvement is taken into account in RESUMMINO for Higgsino, gaugino and slepton pair production $[40,47]$. Since we have given a detailed discussion of the collinear improvement as well as the theoretical status of exponentiating contributions in the $\mathcal{C}$-function [88-90] in a previous publication [71], we do not repeat it here. The same holds for the matching of resummed cross section $\sigma_{a b}^{\text {(res) }}$, valid near threshold, and the normal perturbative calculation $\sigma_{a b}^{\text {(f.o.) }}$, valid outside this region, by adding both results and subtracting the overlap $\sigma_{a b}^{(\exp )}$, i.e., the resummed cross section reexpanded to NNLO. We therefore give here only the contributions to the new coefficient

$$
\mathcal{K}^{(2)}=\mathcal{K}^{(2,1)} L+\mathcal{K}^{(2,2)} L^{2}+\mathcal{K}^{(2,3)} L^{3}+\mathcal{K}^{(2,4)} L^{4}
$$

required at NNLO in the expanded cross section

$$
\begin{aligned}
\sigma_{a b}^{(\exp )}\left(N, M^{2}, \mu_{R}^{2}, \mu_{F}^{2}\right) & =\sigma_{a b}^{(0)} \mathcal{C}_{a b}\left(M^{2}, \mu_{R}^{2}, \mu_{F}^{2}\right) \exp \left[G_{a b}\left(N, M^{2}, \mu_{R}^{2}, \mu_{F}^{2}\right)\right] \\
& =\sigma_{a b}^{(0)}\left[1+\left(\frac{\alpha_{s}}{2 \pi}\right) \mathcal{C}_{a b}^{(1)}+\left(\frac{\alpha_{s}}{2 \pi}\right)^{2} \mathcal{C}_{a b}^{(2)}+\ldots\right]\left[1+\left(\frac{\alpha_{s}}{2 \pi}\right) \mathcal{K}^{(1)}+\left(\frac{\alpha_{s}}{2 \pi}\right)^{2} \mathcal{K}^{(2)}+\ldots\right] \\
& =\sigma_{a b}^{(0)}\left[1+\left(\frac{\alpha_{s}}{2 \pi}\right)\left(\mathcal{C}_{a b}^{(1)}+\mathcal{K}^{(1)}\right)+\left(\frac{\alpha_{s}}{2 \pi}\right)^{2}\left(\mathcal{C}_{a b}^{(2)}+\mathcal{K}^{(2)}+\mathcal{C}_{a b}^{(1)} \mathcal{K}^{(1)}\right)+\ldots\right],
\end{aligned}
$$

which read explicitly $[69,70]$

$$
\begin{gathered}
\mathcal{K}^{(2,1)}=-\frac{C_{F}}{27}\left\{56 n_{f}-404 C_{A}+3 \log \left(\frac{\mu_{F}^{2}}{s}\right)\left[20 n_{f}+2 C_{A}\left(-67+3 \pi^{2}\right)\right.\right. \\
\left.\left.+3\left(11 C_{A}-2 n_{f}\right)\left(\log \left(\frac{\mu_{F}^{2}}{\mu_{R}^{2}}\right)-\log \left(\frac{\mu_{R}^{2}}{s}\right)\right)\right]+378 C_{A} \zeta_{3}\right\}, \\
\mathcal{K}^{(2,2)}=\frac{2}{9} C_{F}\left[-10 n_{f}+67 C_{A}-3 C_{A} \pi^{2}+36 C_{F} \log ^{2}\left(\frac{\mu_{F}^{2}}{s}\right)+\left(33 C_{A}-6 n_{f}\right) \log \left(\frac{\mu_{R}^{2}}{s}\right)\right], \\
\mathcal{K}^{(2,3)}=\frac{4}{9} C_{F}\left[11 C_{A}-2 n_{f}+36 C_{F} \log \left(\frac{\mu_{F}^{2}}{s}\right)\right], \\
\mathcal{K}^{(2,4)}=8 C_{F}^{2} .
\end{gathered}
$$

The SUSY-QCD (squark-gluino loop) corrections are only matched at NLO, since they are not known beyond this order [30]. In this sense, our results are accurate to approximate NNLO (aNNLO) plus NNLL precision. This approximation is justified by the fact that the SUSY-QCD corrections are subdominant due to the large squark and gluino masses. A detailed description of the inverse Mellin transform

$$
M^{2} \frac{d \sigma_{A B}}{d M^{2}}(\tau)=\frac{1}{2 \pi i} \int_{\mathcal{C}_{N}} d N g \tau^{-N} M^{2} \frac{d \sigma_{A B}(N)}{d M^{2}},
$$

that has to be performed for the resummed and the perturbatively expanded results in Mellin space can be found in Ref. [71].

\section{NUMERICAL RESULTS FOR HIGGSINO PAIR PRODUCTION}

Naturalness arguments on the spectrum of SUSY theories require the masses of Higgsinos to be small, i.e., below the TeV scale, and the lightest SUSY particle (LSP) $\tilde{\chi}_{1}^{0}$, the lightest chargino $\left(\tilde{\chi}_{1}^{ \pm}\right)$and the next-to-lightest neutralino $\left(\tilde{\chi}_{2}^{0}\right)$ to be close in mass. Experimental analyses with the largest sensitivity to this kind of compressed scenario consider three main processes, which all lead to signatures with soft leptons and moderate missing transverse momentum in the final state [13]. The first two processes are the associated production of a positively or negatively charged $\tilde{\chi}_{1}^{ \pm}$and a $\tilde{\chi}_{2}^{0}$, while in the third process a pair of charginos $\left(\tilde{\chi}_{1}^{+} \tilde{\chi}_{1}^{-}\right)$is produced. The heavier 
neutralino $\tilde{\chi}_{2}^{0}$ and the charginos $\tilde{\chi}_{1}^{ \pm}$will decay to the lighter $\tilde{\chi}_{1}^{0}$ through an off-shell $Z$ or $W^{ \pm}$boson, respectively. Since the decay products are expected to be soft because of the compressed spectrum, a jet with large transverse momentum produced through initial state radiation (ISR) can enhance the discriminating power with respect to SM processes [13].

Based on an integrated LHC luminosity of $139(36) \mathrm{fb}^{-1}$, the ATLAS (CMS) collaboration have excluded pure, massdegenerate Higgsino pairs $\tilde{\chi}_{1}^{ \pm} \tilde{\chi}_{2}^{0}$ up to 193 (168) GeV, when they decay to 9 (20) GeV lighter $\tilde{\chi}_{1}^{0}$ 's and electroweak $W$ and $Z$ gauge bosons [13,14]. For general gauge-mediated SUSY breaking models, the limits set by the ATLAS collaboration are somewhat stronger with $295 \mathrm{GeV}$ for mass-degenerate Higgsinos including the $\tilde{\chi}_{1}^{0}$ that decay to $Z$ (or $h$ ) bosons and almost massless gravitinos $\tilde{G}$ [15]. In the high-luminosity phase of the LHC (HL-LHC) with $3000 \mathrm{fb}^{-1}$ at a center-ofmass energy $14 \mathrm{TeV}$, the mass reach is expected to extend to $360 \mathrm{GeV}$ [16]. For the invariant-mass distributions we therefore adopt a default $\tilde{\chi}_{2}^{0}\left(\tilde{\chi}_{1}^{ \pm}\right)$mass of $208(203) \mathrm{GeV}$, while for the total cross section analysis we vary the $\tilde{\chi}_{1}^{ \pm}$mass between the LEP limit of $103.5 \mathrm{GeV}$, valid for a mass splitting with the $\tilde{\chi}_{1}^{0}$ of at least $3 \mathrm{GeV}[17,18]$, and $500 \mathrm{GeV}$.

Gluinos enter only at NLO in virtual loop diagrams, so that their masses play a subdominant role. Squarks appear already at LO in the $t$ - and $u$-channel propagators, but since light Higgsinos are mostly produced in the $s$-channel, their masses also have little influence, as does the trilinear coupling $A_{0}$ determining mixing in the sbottom sector. We adopt a squark and gluino mass of $1.3 \mathrm{TeV}$ as our default value, which is still allowed for not too large mass differences with the lightest neutralino, even though the most stringent ATLAS (CMS) mass limits already reach 1.94 (1.63) and 2.35 (2.31) TeV [91,92].

In the following, we compute the cross sections for the aforementioned processes at LO, NLO, NLO + NLL and aNNLO + NNLL adopting CT14 PDFs at LO, NLO and NNLO for consistency [93]. The spectra with the specific characteristics of MSSM scenarios have been obtained with the public code SPheno 4.0.3 [94,95], following the considerations in Ref. [27]. In particular, light Higgsinolike neutralinos and charginos $\tilde{\chi}_{1}^{0}, \tilde{\chi}_{1}^{ \pm}$and $\tilde{\chi}_{2}^{0}$ of masses similar to the Higgsino mass parameter $\mu$ can be obtained by setting this parameter to $\mu \leq M_{1}=M_{2}$, i.e., below the bino and wino mass parameters $M_{1}$ and $M_{2}$. We set $\tan \beta=$ 30 and choose $\mu$ between $100 \mathrm{GeV}$ and $500 \mathrm{GeV}$ in order to stay (not too far) above the experimental exclusion limits, while our choice $M_{1,2}=1 \mathrm{TeV}$ ensures a large Higgsino content and mass splittings of the order of $5 \mathrm{GeV}$ (i.e., $m_{\tilde{\chi}_{2}^{0}}-m_{\tilde{\chi}_{1}^{ \pm}} \approx m_{\tilde{\chi}_{1}^{ \pm}}-m_{\tilde{\chi}_{1}^{0}} \approx 5 \mathrm{GeV}$ ). Our calculations of differential and total cross sections are performed using RESUMMINO [60] interfaced with LHAPDF6 [96] for the interpolation of the PDF grids. The SM parameters have been chosen according to their current PDG values [97], and $\alpha_{s}\left(\mu_{R}\right)$ is computed in accordance with the corresponding CT14 PDF fit.

\section{A. Invariant-mass distributions}

We begin with the invariant-mass distribution for the associated production of a Higgsino-like lightest chargino and a Higgsino-like second-lightest neutralino. These differential cross sections at LO (yellow), NLO (green), NLO + NLL (blue) and aNNLO + NLL (red curve) are shown in the upper panel of Fig. 1. On a logarithmic scale, the uncertainties (shaded bands) coming from variations of the renormalization and factorization scales with the sevenpoint method (i.e., by relative factors of two, but not four) about their central value, the average produced SUSY particle mass, are barely visible, and we will study them in more detail in Fig. 2 below. Also the relative impact of the higher order corrections is only clearly visible with respect to the $\mathrm{LO}$ prediction, so that we have included a lower panel showing the ratios ( $K$ factors) of aNNLO + NLL over NLO (red), NLO + NLL over NLO (green), and also aNNLO + NNLL over NLO + NLL (blue curve) differential cross sections. While the NLO corrections have long been known to enhance the LO cross section by about $30 \%$ [30], the NLL and NNLL corrections increase the NLO cross section by another $3-5 \%$ and $\pm 2 \%$, respectively, showing a good convergence of the perturbative series.

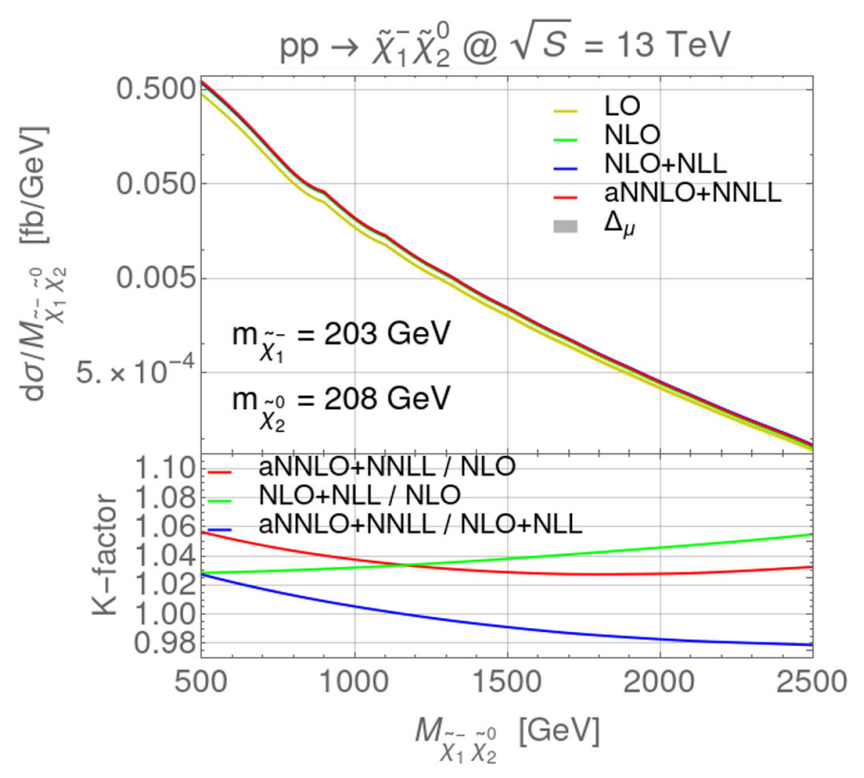

FIG. 1. Top: invariant-mass distribution for the associated production of charginos and neutralinos with masses of $203 \mathrm{GeV}$ and $208 \mathrm{GeV}$ at the LHC with a center-of-mass energy of $\sqrt{S}=13 \mathrm{TeV}$. Shown are results at LO (yellow), NLO (green), NLO + NLL (blue), and aNNLO + NNLL (red) together with the corresponding scale uncertainties (shaded bands). Bottom: ratios ( $K$ factors) of aNNLO + NNLL over NLO (red), NLO + NLL over NLO (green), and aNNLO + NNLL over NLO + NLL (blue) differential cross sections as a function of the invariant mass of the Higgsino pair. 


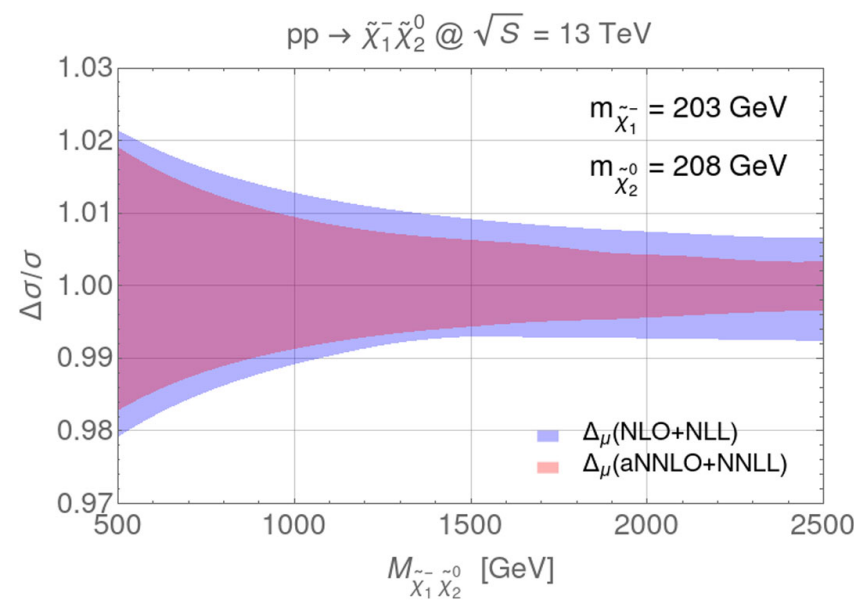

FIG. 2. Scale uncertainty of the invariant-mass distribution in Fig. 1. Shown are the results at NLO + NLL (blue) and aNNLO + NNLL (red shaded band).

This is also demonstrated by the width of the combined scale uncertainty, shown in Fig. 2 as shaded bands at $\mathrm{NLO}+\mathrm{NLL}$ (blue) and aNNLO + NNLL (red). At small and large invariant masses, this uncertainty shrinks from \pm 2.1 to $1.8 \%$ and from \pm 0.6 to $0.4 \%$. As expected, resummation of large threshold logarithms stabilizes the cross section more for large invariant masses, in particular when the final state is mostly produced in the $s$-channel as it is the case for light Higgsinos and sleptons [71].

\section{B. Total cross sections}

The total cross section for the associated production of Higgsino-like charginos and neutralinos is shown in the upper panel of Fig. 3 at LO (yellow), NLO (green), NLO + NLL (blue), and aNNLO + NLL (red curve). Again, only

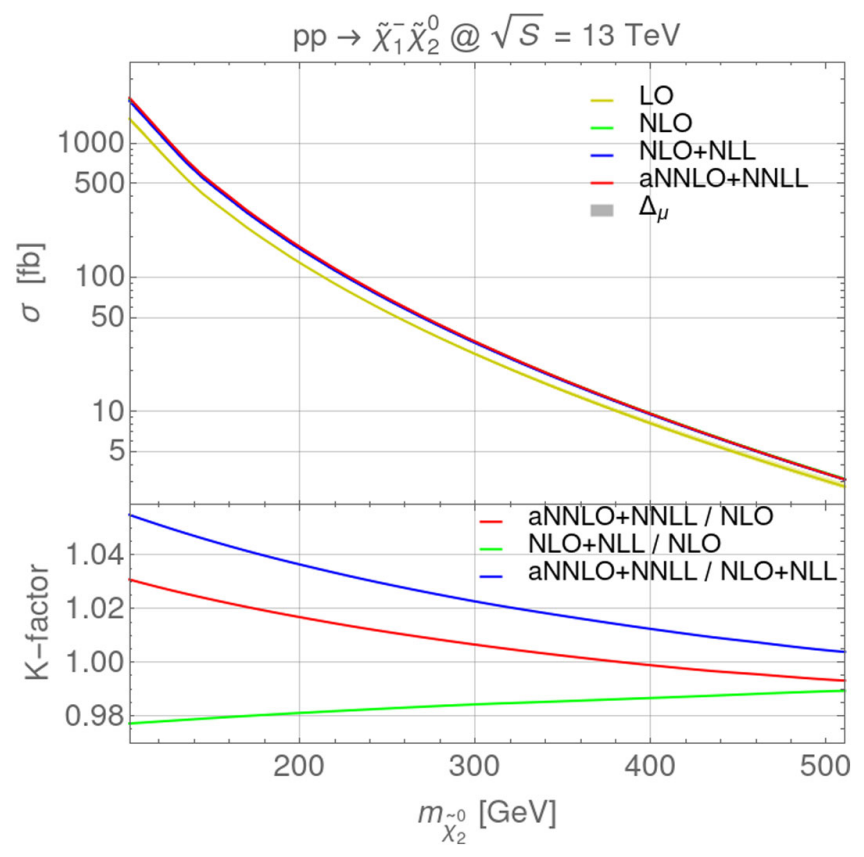

FIG. 3. Top: total cross section for Higgsino-like charginos and neutralinos at the LHC with a center-of-mass energy of $\sqrt{S}=$ $13 \mathrm{TeV}$ as a function of the $\tilde{\chi}_{2}^{0}$ mass. Shown are results at LO (yellow), NLO (green), NLO + NLL (blue), and aNNLO + NNLL (red) together with the corresponding scale uncertainties (shaded bands). Bottom: ratios ( $K$ factors) of aNNLO + NNLL over NLO (red), NLO + NLL over NLO (green), and aNNLO + NNLL over NLO + NLL (blue) total cross sections.

the increase from LO to higher order is clearly visible on the logarithmic scale. The enhancements from NLO to aNNLO + NNLL (red) and to NLO + NLL (green) as well as their ratio (blue) are therefore shown in the lower panel. The aNNLO + NNLL corrections increase the total cross
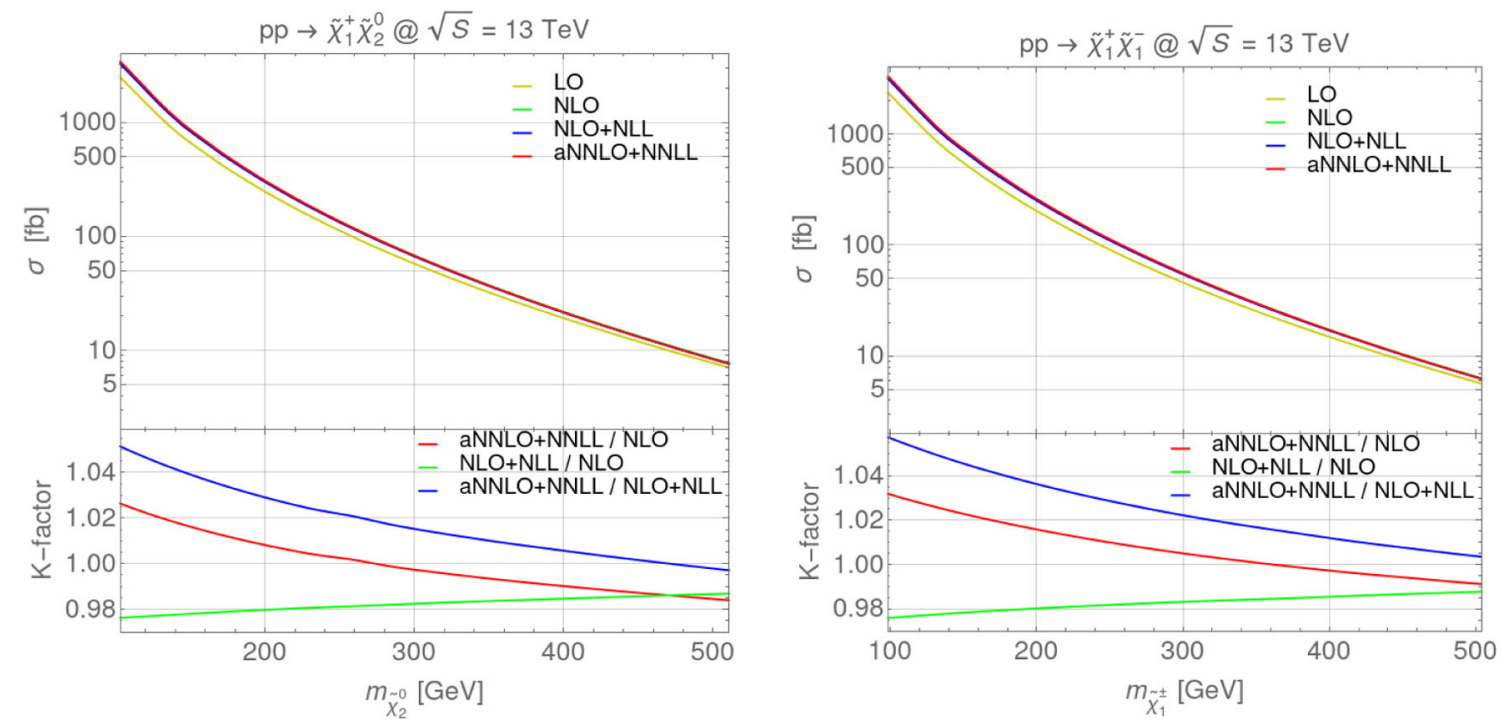

FIG. 4. Same as Fig. 3, but for the associated production of a positively charged Higgsino with the second-lightest neutralino (left) and for the pair production of charginos (right). 

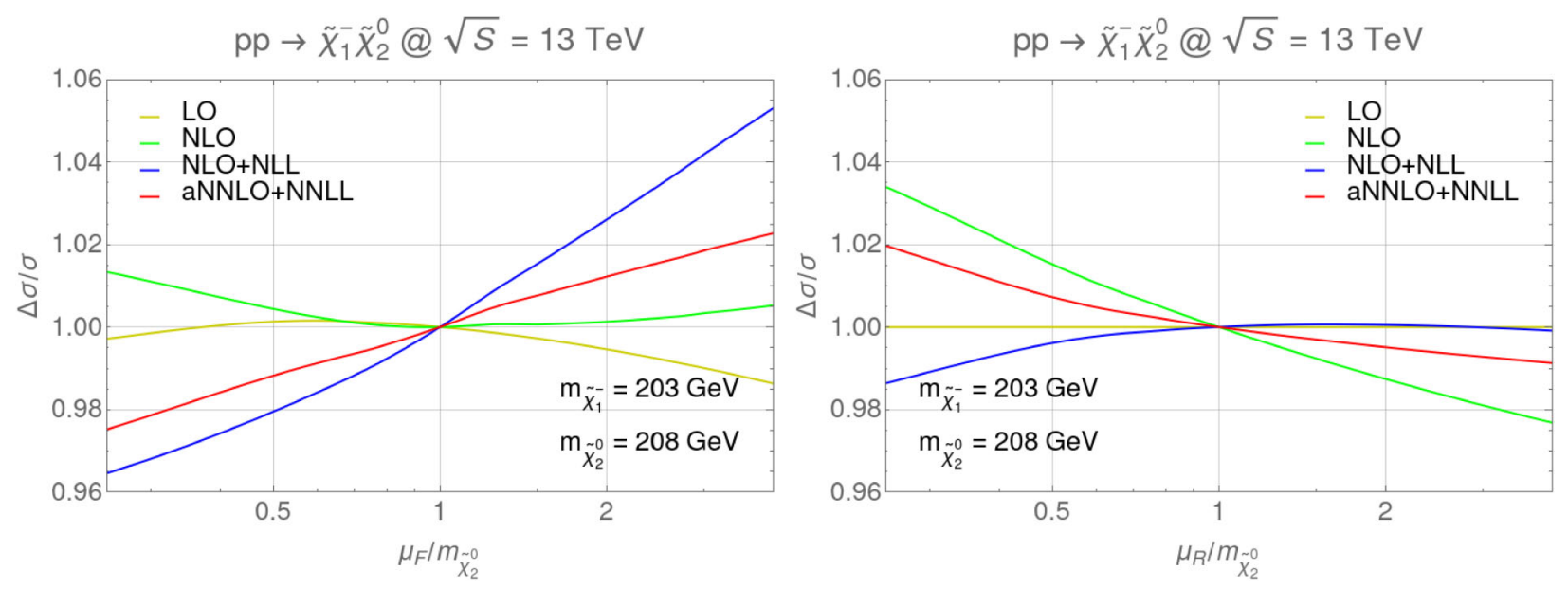

FIG. 5. Relative variation of the total cross section for Higgsino-like chargino-neutralino pairs as a function of the factorization (top) and renormalization scale (bottom). Shown are results at LO (yellow), NLO (green), NLO + NLL (blue), and aNNLO + NNLL (red).

section by up to $5 \%$ for low Higgsino masses, and the perturbation series converges nicely for large Higgsino masses.

The situation is very similar for the production of Higgsino-like $\tilde{\chi}_{1}^{+} \tilde{\chi}_{2}^{0}$ and chargino pairs $\tilde{\chi}_{1}^{+} \tilde{\chi}_{1}^{-}$, shown in Fig. 4. The main difference is the absolute size of the total cross section, which at a $p p$ collider like the LHC is largest for positively charged final states, followed by neutral and negatively charged final states. The magnitude of the difference depends on the $x$-range probed in the PDFs and thus on the Higgsino masses. It increases toward larger masses, where valence quarks play a more important role.

The dependence of the total Higgsino cross section on the factorization (top) and renormalization (bottom) scales is studied individually in Fig. 5. While the latter is only introduced only at NLO (green), the former includes a weak dependence from the PDFs already at LO (yellow). From
$\mathrm{NLO}+\mathrm{NLL}$ (blue) to aNNLO + NNLL (red) one observes a reduction in particular for the factorization scale. At these relatively low Higgsino masses of 203 and $208 \mathrm{GeV}$, respectively, both uncertainties still amount to about $\pm 2 \%$, while at NLO and even NLO + NLL they could still reach about $\pm 4 \%$.

This is also reflected in Fig. 6 (left), where both uncertainties are varied with the seven-point method and shown as a function of the Higgsino mass. As expected, the combined uncertainty is reduced for heavier Higgsinos to a level of about $\pm 2 \%$ at NLO + NLL (blue) and only $\pm 0.5 \%$ at aNNLO + NNLL (red). The situation for Higgsinos, which are mostly in the $s$-channel, is thus similar to the one for sleptons [71]. For comparison, Fig. 6 (right) shows the scale uncertainties at NLO (yellow), aNLO (red), and aNNLO (green shaded band). While the scale uncertainty at NLO depends only weakly on the Higgsino mass, the
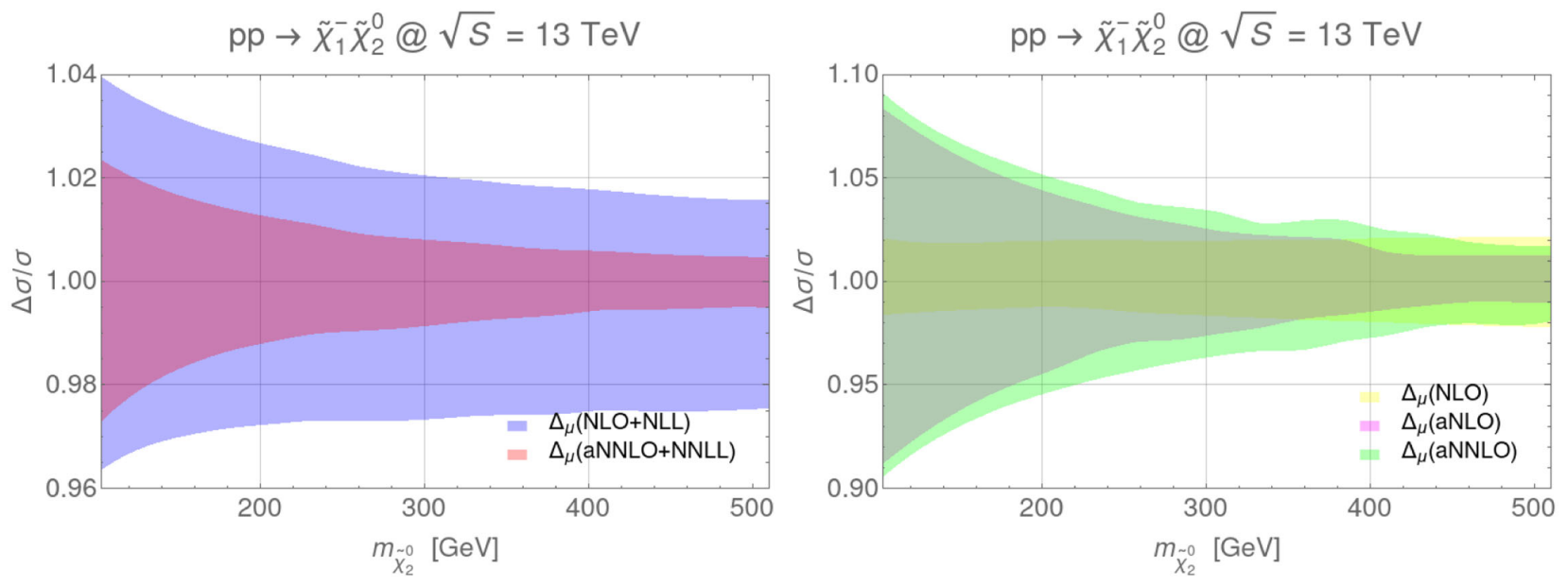

FIG. 6. Scale uncertainty of the total cross section for Higgsino-like chargino-neutralino pairs at the LHC with a center-of-mass energy of $\sqrt{S}=13 \mathrm{TeV}$ as a function of the neutralino mass. Left: results at NLO + NLL (blue) and aNNLO + NNLL (red). Right: results at NLO (yellow), aNLO (red), and aNNLO (green shaded band). 


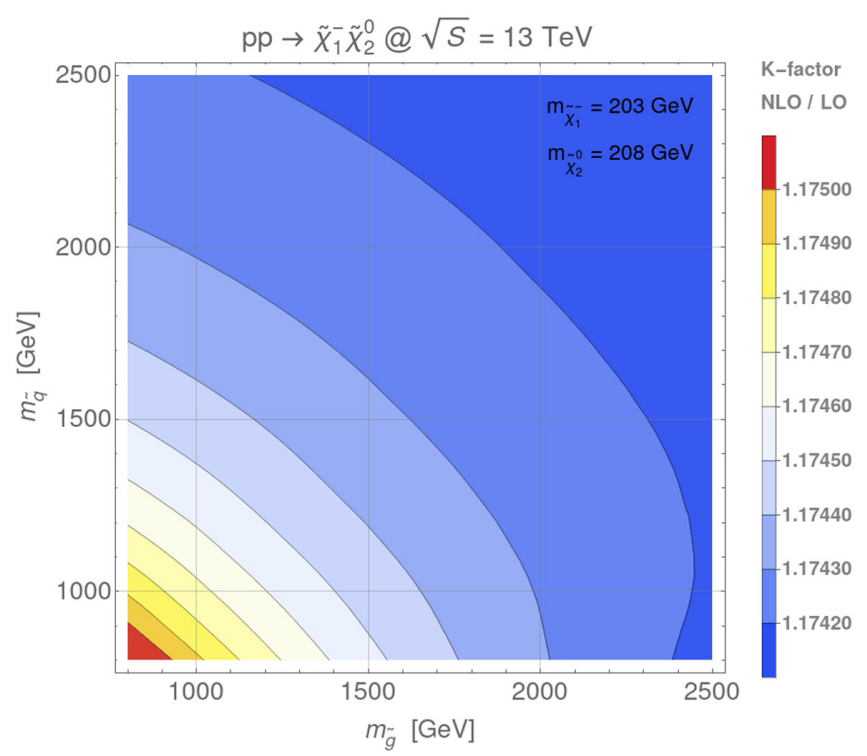

FIG. 7. Ratio ( $K$ factor) of NLO over LO total cross sections (both with NLO PDFs) for Higgsino pair production at the LHC with a center-of-mass energy of $\sqrt{S}=13 \mathrm{TeV}$ as a function of the squark and gluino masses.

approximations at aNLO and aNNLO are clearly only valid for larger Higgsino masses, where their scale dependences approach the one at NLO. This far from threshold, the difference of aNNLO vs aNLO is mainly induced by the use of different PDFs (NNLO vs NLO). Remember that the central $K$-factors also differ due to squark and gluino mass dependent terms not included in the aNLO and aNNLO approximations (see Fig. 3).

Since the $t$ - and $u$-channels play a subdominant role for Higgsinos, not only the dependence on the gluino mass, introduced only at NLO, but also the one on the squark masses should be weak. This can clearly be seen in Fig. 7, where the ratio of the NLO (and similarly any other

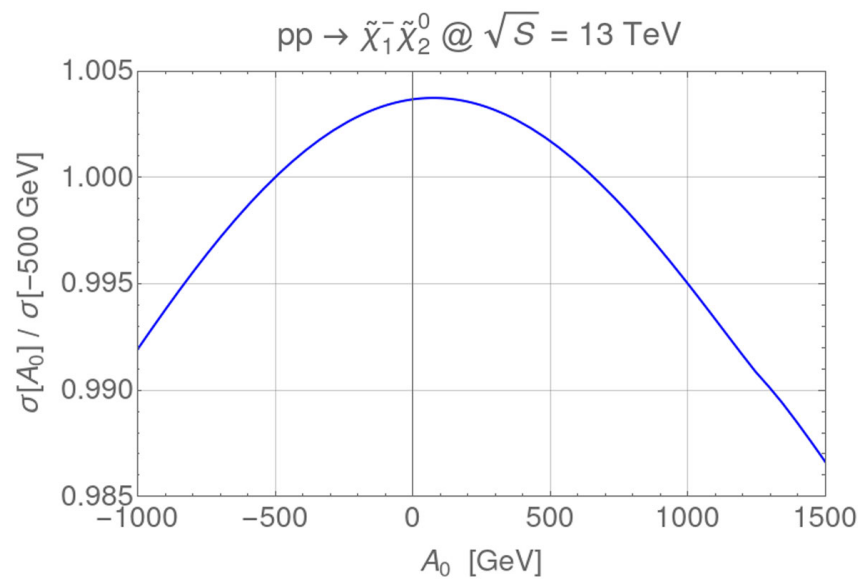

FIG. 8. Dependence of the NLO (or NLO + NLL or aNNLO + NNLL) total cross section on the common trilinear coupling $A_{0}$ that governs squark mixing in the sbottom sector. higher-order) cross section over the LO one is shown in the squark-gluino mass plane. Overall, it varies by less than one per mill. The gradient is along the diagonal and slightly steeper when the squark and gluino masses are still relatively close to those of the Higgsinos.

When the squark masses are not all identified with each other, but mixing in the sbottom sector is allowed, a dependence on the trilinear coupling $A_{0}$ is introduced. It is shown in Fig. 8. As expected, for Higgsinos it is also weak and amounts to at most $+0.4 \%$ and $-1.3 \%$ when compared with the cross section in our default scenario with $A_{0}=-500 \mathrm{GeV}$.

\section{NUMERICAL RESULTS FOR GAUGINO PAIR PRODUCTION}

We now turn to the case where the produced neutralinos and charginos have a large gaugino component. The next-tolightest neutralino $\tilde{\chi}_{2}^{0}$ and the charginos $\tilde{\chi}_{1}^{ \pm}$will be considered as winolike and almost degenerate with a mass above $1100 \mathrm{GeV}$ to satisfy experimental constraints, while the LSP $\tilde{\chi}_{1}^{0}$ is assumed to be binolike and light. In this scenario, large production cross sections of $\tilde{\chi}_{1}^{ \pm} \tilde{\chi}_{2}^{0}$ and short decay chains are expected. For example, assuming an intermediate and equal mass for left-handed staus and tau sneutrinos, the winos will decay through these states into the LSP, taus, and tau neutrinos, leading to interesting collider signatures [98]. This particular spectrum of particle masses can be achieved within the phenomenological MSSM (pMSSM) framework. It is of particular interest, since the coannihilation of light staus with the LSP can generate a dark matter relic density in accordance with the observations [5,12].

Based on an integrated LHC luminosity of $36 \mathrm{fb}^{-1}$, the ATLAS (CMS) collaboration have excluded pure, massdegenerate wino pairs $\tilde{\chi}_{1}^{ \pm} \tilde{\chi}_{2}^{0}\left(\tilde{\chi}_{1}^{+} \tilde{\chi}_{1}^{-}\right)$decaying with $100 \%$ branching ratio via sleptons to significantly lighter pure binos $\tilde{\chi}_{1}^{0}$ up to masses of 1100 (800) $\mathrm{GeV}[19,20]$. With $139 \mathrm{fb}^{-1}$, the ATLAS collaboration could also exclude chargino pairs up to masses of $1000 \mathrm{GeV}$ [21]. For pure winos decaying to on-shell gauge and Higgs bosons, the ATLAS (CMS) limits are sometimes considerably weaker and reach only 345 to 1000 (650) GeV, depending on the analysis method and despite luminosities of up to $139 \mathrm{fb}^{-1}$ [21-24].

The dependence on the gluino mass, which enters only at NLO, is again expected to be weak. However, the squark mass dependence will now be more important, as heavy gauginos can have large LO contributions from $t$ - and $u$-channel diagrams and their (negative) interferences with the $s$-channel. In addition, hadronic gaugino decay channels will be open when $m_{\tilde{q}}<m_{\tilde{\chi}_{1}^{ \pm}} \tilde{\mathcal{X}}_{2}^{0}$, and squark threshold effects will appear in the one-loop diagrams when $m_{\tilde{\chi}_{1}^{ \pm}, \tilde{\chi}_{2}^{0}} \simeq m_{\tilde{q}}$. These thresholds will also affect the dependence on the trilinear coupling $A_{0}$ controlling the physical sbottom masses. 
Our desired SUSY spectrum with winolike charginos and neutralinos and a binolike LSP is obtained using again the public code SPHENO 4.0.3 [94,95] and by choosing a small value for the bino mass parameter $M_{1}=100 \mathrm{GeV}$, while the wino mass parameter $M_{2}>1 \mathrm{TeV}$ is chosen above the ATLAS exclusion limits. The large gaugino content can be achieved by choosing a large value for $\mu=3 \mathrm{TeV} \gg M_{2}$. With this configuration, only a very small splitting between the masses of the neutralino $\tilde{\chi}_{2}^{0}$ and the charginos $\tilde{\chi}_{1}^{ \pm}$is generated, while the LSP $\tilde{\chi}_{1}^{0}$ remains light.

\section{A. Invariant-mass distributions}

We begin our discussion with the invariant-mass distribution for the associated production of winolike lightest charginos and second-lightest neutralinos. These differential cross sections at LO (yellow), NLO (green), NLO + NLL (blue), and aNNLO + NLL (red curve) are shown in the upper panel of Fig. 9. In contrast to the Higgsino case, the NLO corrections increase the LO cross section only at low invariant masses, but decrease it for large invariant masses. A decrease for all invariant masses is observed from NLO + NLL to aNNLO + NNLL (see also the lower panel). This behavior is correlated with large $t$ - and $u$-channel contributions and large cancellations of the squared $s$-channel contribution with its interference terms.

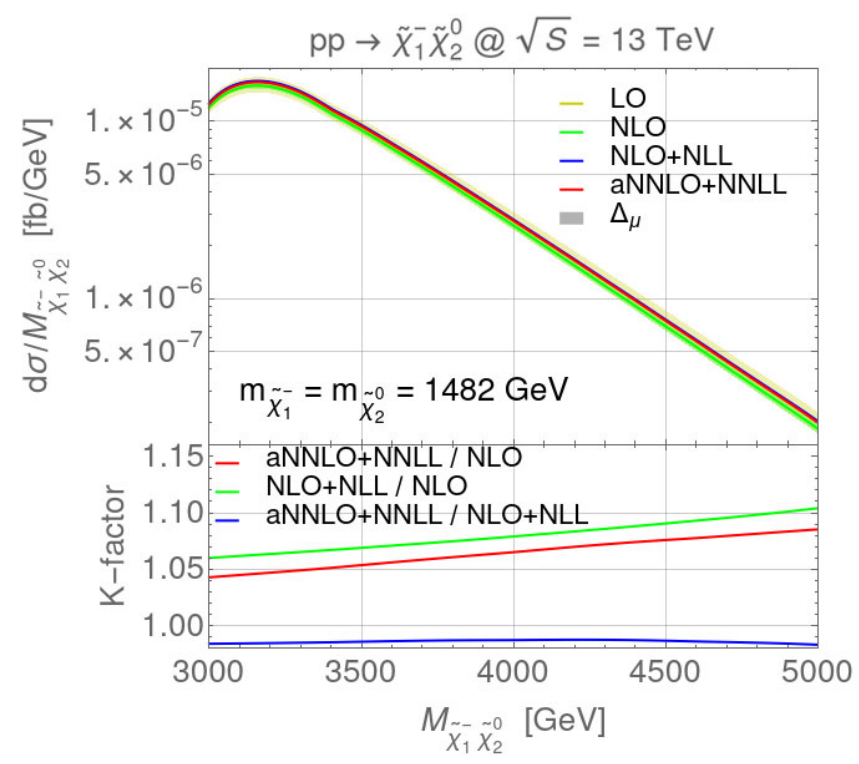

FIG. 9. Top: invariant-mass distribution for the associated production of charginos and neutralinos with masses of $1482 \mathrm{GeV}$ at the LHC with a center-of-mass energy of $\sqrt{S}=13 \mathrm{TeV}$. Shown are results at LO (yellow), NLO (green), $\mathrm{NLO}+\mathrm{NLL}$ (blue), and aNNLO + NNLL (red) together with the corresponding scale uncertainties (shaded bands). Bottom: ratios ( $K$ factors) of aNNLO + NNLL over NLO (red), NLO + NLL over NLO (green), and aNNLO + NNLL over NLO + NLL (blue) differential cross sections as a function of the invariant mass of the gaugino pair.

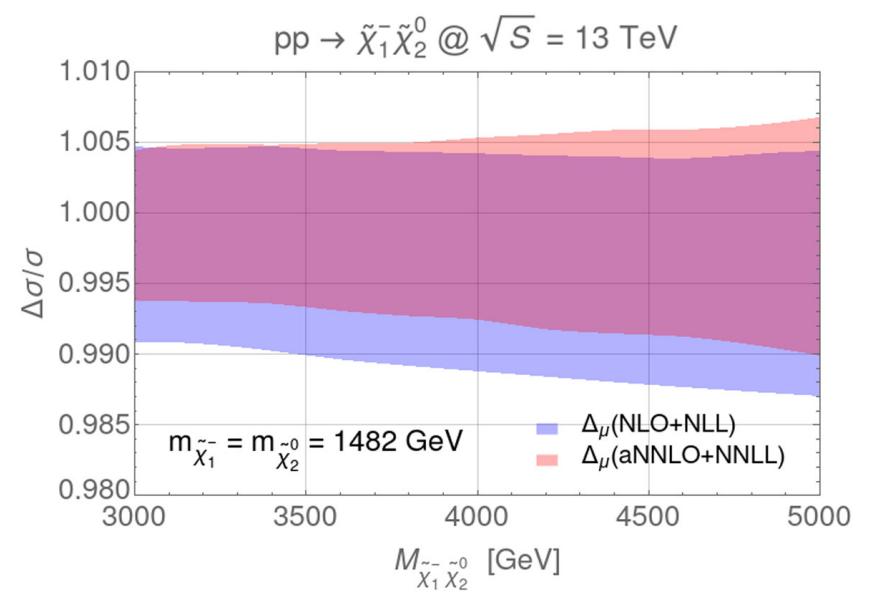

FIG. 10. Scale uncertainty of the invariant-mass distribution in Fig. 9. Shown are the results at NLO + NLL (blue) and aNNLO + NNLL (red shaded band).

The combined scale uncertainty for this distribution is shown in Fig. 10 at NLO + NLL (blue) and aNNLO + NNLL (red). A reduction from $\pm 0.7 \%$ to $\pm 0.5 \%$ is observed at low invariant masses. The reduction is smaller for large invariant masses, which is again related to the importance of the $t$ - and $u$-channels.

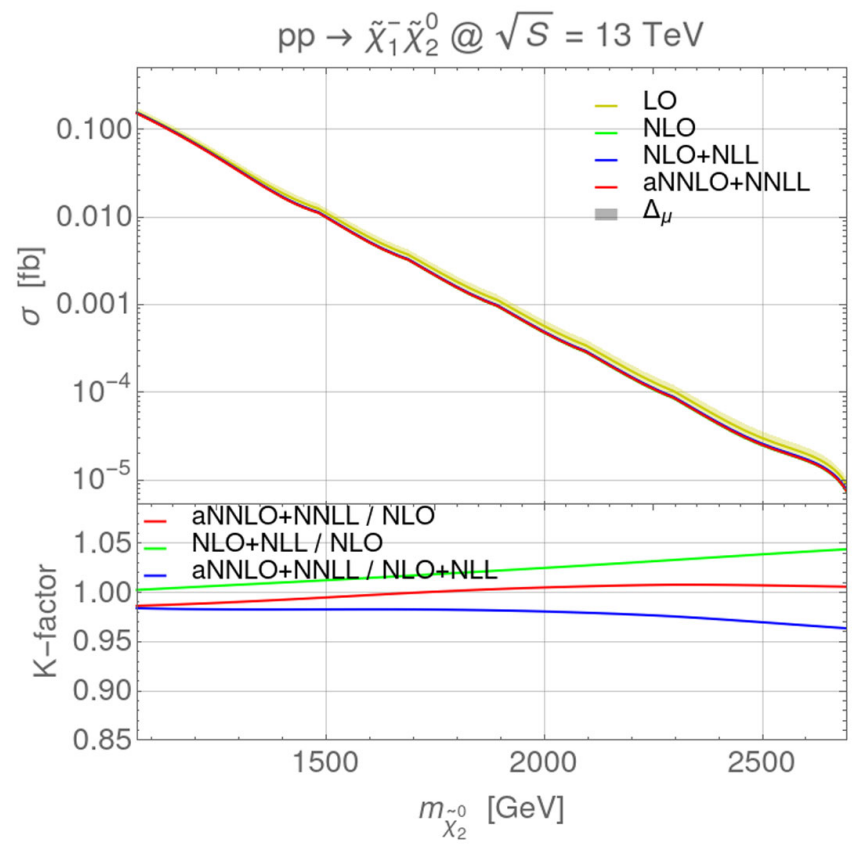

FIG. 11. Top: total cross section for gaugino-like charginos and neutralinos at the LHC with a center-of-mass energy of $\sqrt{S}=$ $13 \mathrm{TeV}$ as a function of the $\tilde{\chi}_{2}^{0}$ mass. Shown are results at LO (yellow), NLO (green), NLO + NLL (blue), and aNNLO + NNLL (red) together with the corresponding scale uncertainties (shaded bands). Bottom: ratios ( $K$ factors) of aNNLO + NNLL over NLO (red), NLO + NLL over NLO (green), and aNNLO + NNLL over NLO + NLL (blue) total cross sections. 

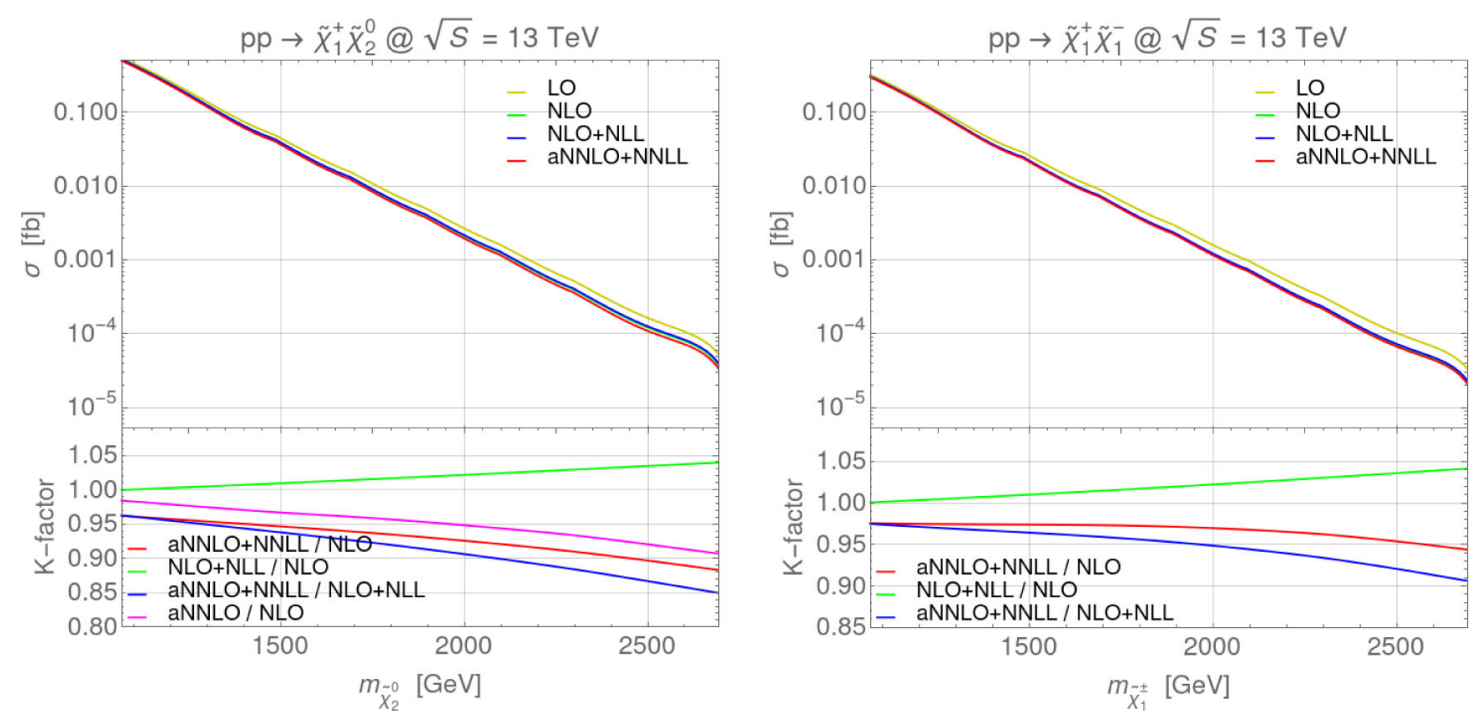

FIG. 12. Same as Fig. 11, but for the associated production of a positively charged gaugino with the second-lightest neutralinos (left) and for the pair production of charginos (right). For the former, we also show the aNNLO/NLO $K$-factor (purple), i.e., without resummation effects.

\section{B. Total cross sections}

We now turn to the total cross sections for gauginos. They are shown in Fig. 11 for the associated production of a negatively charged and a neutral wino as a function of the second-lightest neutralino (and lightest chargino) mass. For our choice of parameters and after integration over the invariant mass, the NLO prediction is smaller than the LO one over the entire $\tilde{\chi}_{2}^{0}$ mass range, and the size of the aNNLO + NNLL corrections is very small in this particular case.

This is, however, a peculiarity of the chosen channel with a negative chargino, as can be seen from Fig. 12 showing the total cross sections for the associated production of a positive chargino with a neutralino (left) and for chargino pair production (right). Both the absolute size of the cross

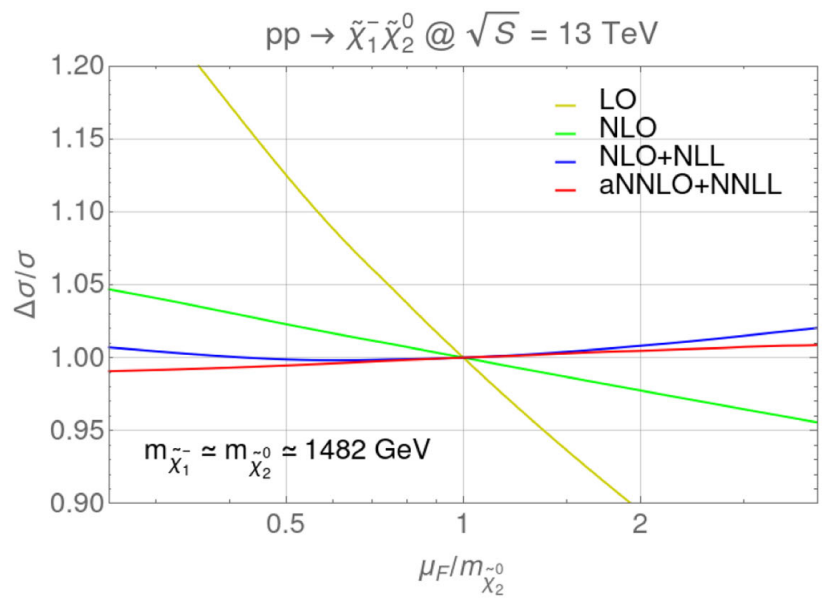

section and the size of the corrections are then different due to the fact that we probe large momentum fractions $x$ and therefore the valence quark structure in the PDFs. In particular, the cross section for $\tilde{\chi}_{1}^{+} \tilde{\chi}_{2}^{0}$ is larger than the one for $\tilde{\chi}_{1}^{-} \tilde{\chi}_{2}^{0}$ by about a factor of four, and the aNNLO + NNLL corrections now amount to up to -12 to $-15 \%$ with respect to the NLO and NLO + NLL predictions. The cross section for chargino pair production through a neutral current represents an intermediate case, as expected. For the case of $\tilde{\chi}_{1}^{+} \tilde{\chi}_{2}^{0}$ production, we also show the aNNLO/ NLO $K$-factor (purple), i.e., without including resummation effects. Apart from a small overall shift in the normalization, it is very similar to the one including the NNLL terms. This confirms that the main effect of resumming the higher order terms is not an additional

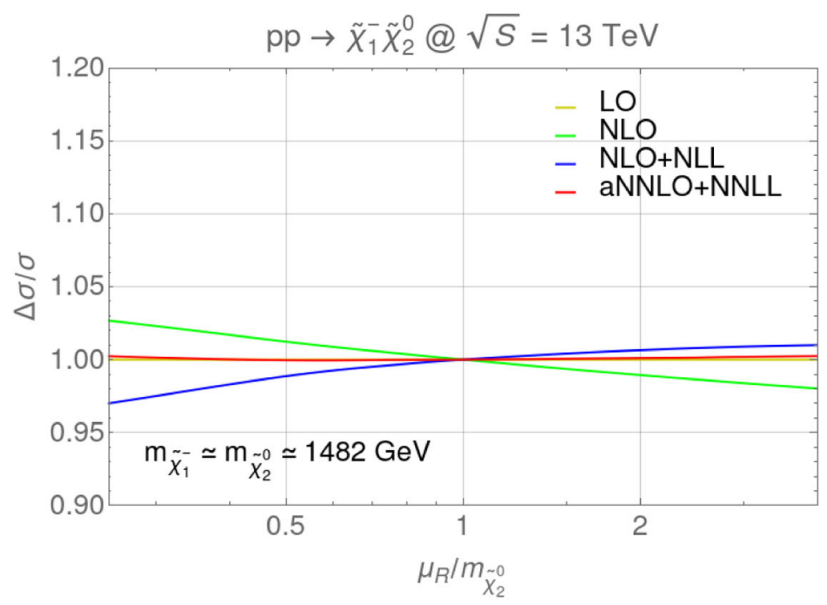

FIG. 13. Relative variation of the total cross section for gauginolike chargino-neutralino pairs as a function of the factorization (top) and renormalization scale (bottom). Shown are results at LO (yellow), NLO (green), NLO + NLL (blue), and aNNLO + NNLL (red). 

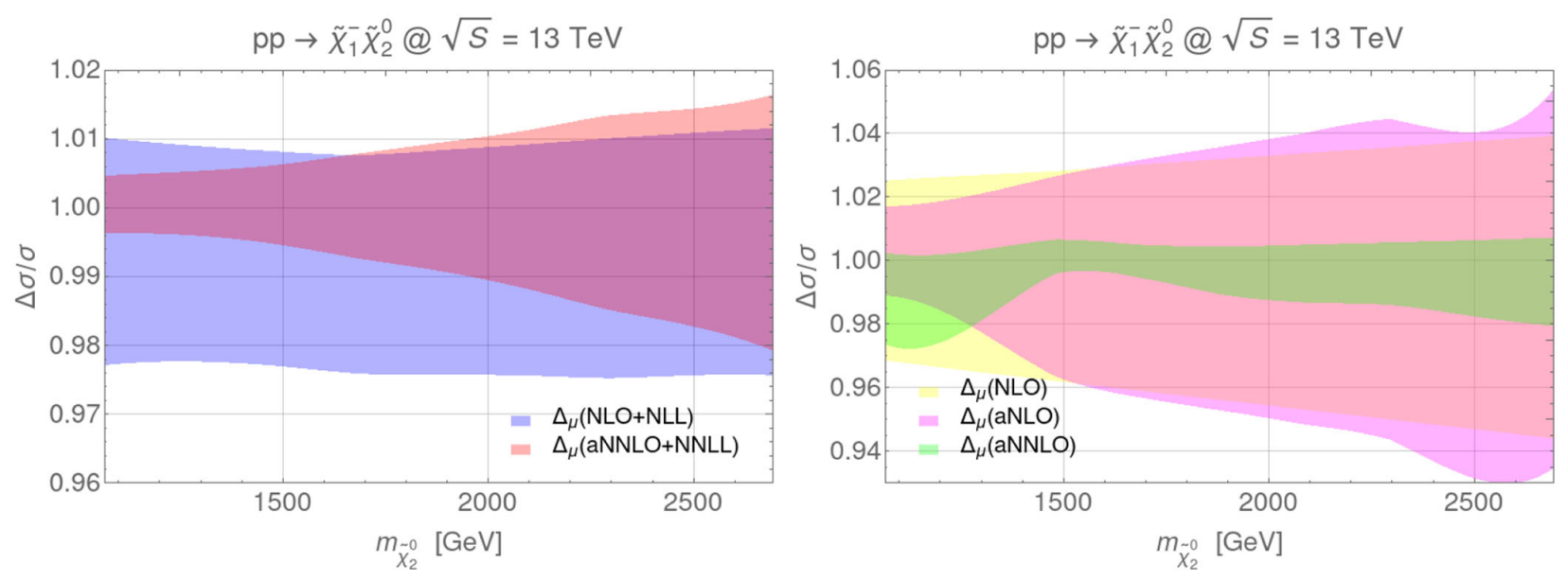

FIG. 14. Scale uncertainty of the total cross section for gauginolike chargino-neutralino pairs at the LHC with a center-of-mass energy of $\sqrt{S}=13 \mathrm{TeV}$ as a function of the neutralino mass. Left: results at NLO + NLL (blue) and aNNLO + NNLL (red). Right: results at NLO (yellow), aNLO (red), and aNNLO (green shaded band).

large shift in the cross section, but its further stabilization with respect to scale uncertainties and corresponds to previous findings in other channels.

The dependence of the total gaugino cross section on the factorization (top) and renormalization (bottom) scale is studied individually in Fig. 13. As in the Higgsino case, the $\mathrm{LO}$ cross section is independent of the renormalization scale. The dependence introduced at NLO of $5 \%$ is reduced at $\mathrm{NLO}+\mathrm{NLL}$ to $4 \%$ and to below percent level at aNNLO+ NNLL. A similarly impressive reduction has been observed for sleptons [71]. The LO factorization scale dependence is much stronger than in the Higgsino case, as we are probing the evolution of the PDFs from the GeV- to the TeV-region. It is reduced from more than $30 \%$ at $\mathrm{LO}$ to $10 \%$ at NLO, then to $2 \%$ at NLO + NLL and aNNLO + NNLL.

In Fig. 11 we observed a better convergence of the perturbative series for not too heavy gauginos than for very large masses. This behavior is reflected in Fig. 14 (left), where the total scale uncertainty also increases toward very large gaugino masses. At $1.1 \mathrm{TeV}$, it amounts to $3 \%$ at $\mathrm{NLO}+\mathrm{NLL}$ (blue) and only $1 \%$ at aNNLO + NNLL (red), while at $2.7 \mathrm{TeV}$ it amounts to $3 \%$ in both cases. For comparison, Fig. 14 (right) shows the scale uncertainties at NLO (yellow), aNLO (red), and aNNLO (green shaded band). As for Higgsinos, the scale uncertainty at NLO depends only weakly on the gaugino mass, but as it is much closer to threshold, the approximation at aNLO works now relatively well over the entire mass range shown, and the aNNLO approximation exhibits an additional reduction.

Figure 15 shows the dependence of the NLO/LO $K$-factor for the production of gauginolike charginos and neutralinos on the squark and gluino masses. As expected, the dependence on the gluino mass, which enters only at NLO, is indeed weak and almost invisible, when the squark mass differs substantially from the gaugino mass of about 1.5 TeV. In contrast, when the squark mass is close to the gaugino mass, the squark mass has a substantial influence already at tree-level, but also at NLO (and beyond), when the squark threshold is crossed in virtual box diagrams. In this situation, also the gluino mass can induce a significant variation of the $K$-factor. The fact that the $\mathrm{NLO} / \mathrm{LO}$ cross section ratio can reach values much larger than one is related to the (almost) on-shell production of intermediate squarks in the final state that subsequently decay into the observed gauginos. This situation therefore requires a careful identification of squark and gaugino production, respectively, from the observed decay products and in particular the presence of jets. We have verified that the relative dependence on squark and gluino masses is

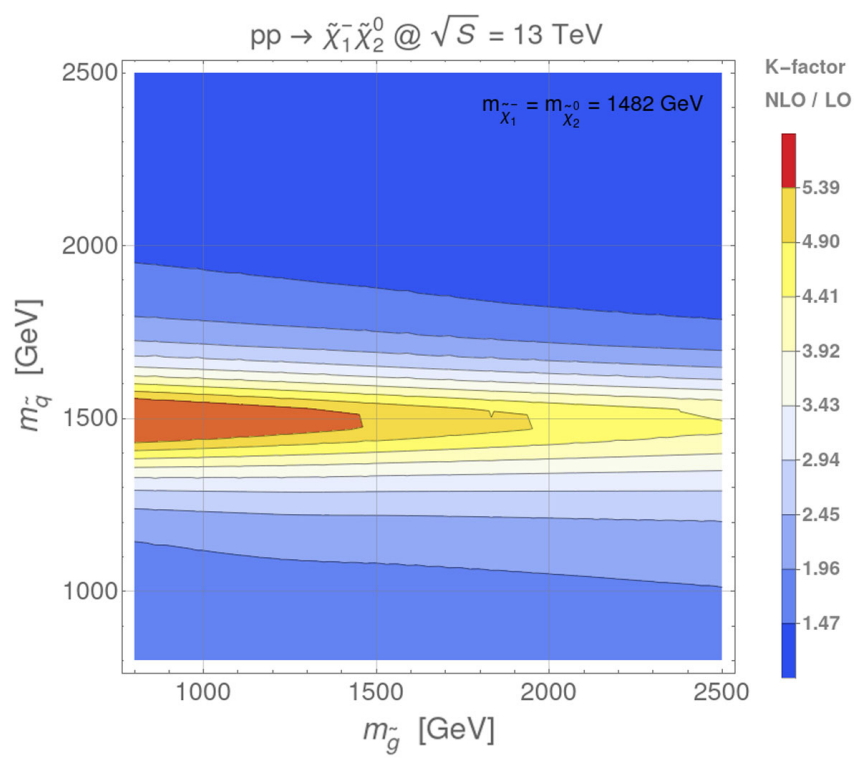

FIG. 15. Ratio ( $K$ factor) of NLO over LO total cross sections (both with NLO PDFs) for gaugino pair production at the LHC with a center-of-mass energy of $\sqrt{S}=13 \mathrm{TeV}$ as a function of the squark and gluino masses. 


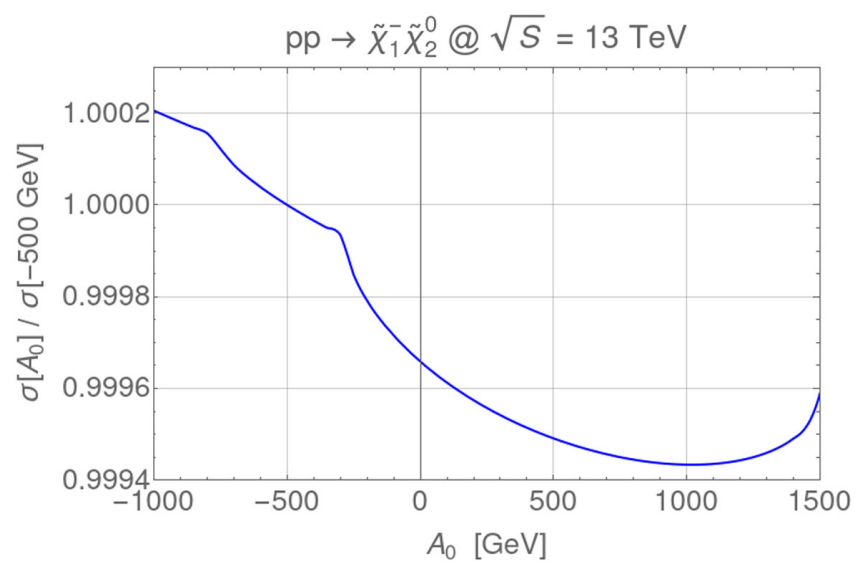

FIG. 16. Dependence of the NLO (or NLO + NLL or aNNLO + NNLL) total cross section on the common trilinear coupling $A_{0}$ that governs squark mixing in the sbottom sector. Shown is the ratio over the default scenario with $A_{0}=-500 \mathrm{GeV}$.

identical for positive charginos. Only the absolute range of the $K$-factor is slightly reduced and ranges then from 1.68 to 5.04, due to the larger LO cross section for positive charginos.

The presence of squark thresholds is also observed in Fig. 16 for bottom squarks. This figure shows the dependence of the NLO (or NLO + NLL or aNNLO + NNLL) total gaugino cross section on the trilinear coupling $A_{0}$ over its value for our default choice of $A_{0}=-500 \mathrm{GeV}$. While the overall dependence is very weak, as bottom quarks in the proton PDFs contribute very little to the total cross section at these large values of $x$, the kinks when the two physical sbottom mass thresholds are crossed are nevertheless clearly visible at $A_{0}=-800 \mathrm{GeV}$ and $A_{0}=-300 \mathrm{GeV}$.

\section{CONCLUSION}

In conclusion, we have presented in this paper the improvement of our previous predictions for Higgsino and gaugino pair production at the LHC from NLO + NLL to aNNLO + NNLL precision. We have briefly reviewed the formalism employed for the threshold resummation of large logarithms that can spoil the convergence of the perturbative series and highlighted the analytical results required for the resummation at NNLL accuracy and its matching to the fixed order calculation at aNNLO. Numerical results were presented for two very different scenarios, i.e., Higgsino and gaugino pair production at the LHC. The mass limits on Higgsinos from the LHC are still relatively weak, they can thus still be as light as a (few) hundred $\mathrm{GeV}$ and consequently produced mostly in the $s$-channel. The aNNLO + NNLL results were found to induce only small modifications of the differential and total cross sections and to stabilize them even more than before at NLO + NLL with respect to variations of the factorization and renormalization scales. For gauginos, which like squarks and gluinos have recently been constrained by LHC searches to the $\mathrm{TeV}$ region and beyond, also $t$ - and $u$-channels and thus the dependence on the squark mass became important already at tree-level, and the impact of the higher-order corrections in the large $x$-region required a closer look. It varied not only with the considered production channel, i.e., the total charge of the final state, but also with the squark mass and, in the threshold region, even the gluino mass. As an additional new aspect, we included in our calculation explicitly the mixing in the squark sector, which proved to be relevant in practice only for bottom (s) quarks and thus more for light Higgsinos produced from partons at small $x$ than for heavier gauginos produced from partons at larger values of $x$. The new results described in this paper will be made publicly available in the next release of RESUMMINO.

\section{ACKNOWLEDGMENTS}

This work has been supported by the BMBF under Contract No. 05H18PMCC1.
[1] H. P. Nilles, Phys. Rep. 110, 1 (1984).

[2] H. E. Haber and G. L. Kane, Phys. Rep. 117, 75 (1985).

[3] J. A. Aguilar-Saavedra et al., Eur. Phys. J. C 46, 43 (2006).

[4] J. R. Ellis, J. Hagelin, D. V. Nanopoulos, K. A. Olive, and M. Srednicki, Nucl. Phys. B238, 453 (1984).

[5] J. R. Ellis, T. Falk, K. A. Olive, and M. Srednicki, Astropart. Phys. 13, 181 (2000); 15, 413(E) (2001).

[6] M. Klasen, M. Pohl, and G. Sigl, Prog. Part. Nucl. Phys. 85, 1 (2015).

[7] B. Herrmann, M. Klasen, and K. Kovarik, Phys. Rev. D 79, 061701 (2009).
[8] B. Herrmann, M. Klasen, and K. Kovarik, Phys. Rev. D 80, 085025 (2009).

[9] J. Harz, B. Herrmann, M. Klasen, K. Kovarik, and Q. Boulc'h, Phys. Rev. D 87, 054031 (2013).

[10] J. Harz, B. Herrmann, M. Klasen, and K. Kovarik, Phys. Rev. D 91, 034028 (2015).

[11] J. Harz, B. Herrmann, M. Klasen, K. Kovarik, and M. Meinecke, Phys. Rev. D 91, 034012 (2015).

[12] J. Branahl, J. Harz, B. Herrmann, M. Klasen, K. Kovarik, and S. Schmiemann, Phys. Rev. D 100, 115003 (2019). 
[13] A. M. Sirunyan et al. (CMS Collaboration), Phys. Lett. B 782, 440 (2018).

[14] G. Aad et al. (ATLAS Collaboration), Phys. Rev. D 101, 052005 (2020).

[15] M. Aaboud et al. (ATLAS Collaboration), Phys. Rev. D 98, 032009 (2018).

[16] CMS Collaboration, Searches for light higgsino-like charginos and neutralinos at the HL-LHC with the Phase-2 CMS detector, Report No. CMS-PAS-FTR-18-001, 2018.

[17] A. Heister et al. (ALEPH Collaboration), Phys. Lett. B 533, 223 (2002).

[18] J. Abdallah et al. (DELPHI Collaboration), Eur. Phys. J. C 31, 421 (2003).

[19] M. Aaboud et al. (ATLAS Collaboration), Eur. Phys. J. C 78, 995 (2018).

[20] A. M. Sirunyan et al. (CMS Collaboration), J. High Energy Phys. 11 (2018) 079.

[21] G. Aad et al. (ATLAS Collaboration), Eur. Phys. J. C 80, 123 (2020).

[22] G. Aad et al. (ATLAS Collaboration), Phys. Rev. D 101, 072001 (2020).

[23] G. Aad et al. (ATLAS Collaboration), Eur. Phys. J. C 80, 691 (2020).

[24] A. Sirunyan et al. (CMS Collaboration), J. High Energy Phys. 03 (2018) 160.

[25] J. Alwall, P. Schuster, and N. Toro, Phys. Rev. D 79, 075020 (2009).

[26] L. Calibbi, J. M. Lindert, T. Ota, and Y. Takanishi, J. High Energy Phys. 11 (2014) 106.

[27] B. Fuks, M. Klasen, S. Schmiemann, and M. Sunder, Eur. Phys. J. C 78, 209 (2018).

[28] W. Beenakker, R. Hopker, M. Spira, and P. M. Zerwas, Nucl. Phys. B492, 51 (1997).

[29] W. Beenakker, M. Kramer, T. Plehn, M. Spira, and P. M. Zerwas, Nucl. Phys. B515, 3 (1998).

[30] W. Beenakker, M. Klasen, M. Kramer, T. Plehn, M. Spira, and P. M. Zerwas, Phys. Rev. Lett. 83, 3780 (1999); 100, 029901(E) (2008).

[31] E. L. Berger, M. Klasen, and T. M. Tait, Phys. Lett. B 459, 165 (1999).

[32] E. L. Berger, M. Klasen, and T. M. Tait, Phys. Rev. D 62 , 095014 (2000).

[33] M. Spira, in 10th International Conference on Supersymmetry and Unification of Fundamental Interactions (SUSY02) (DESY, Hamburg, Germany, 2002), pp. 217-226.

[34] L. G. Jin, C. S. Li, and J. J. Liu, Phys. Lett. B 561, 135 (2003).

[35] T. Binoth, D. Goncalves Netto, D. Lopez-Val, K. Mawatari, T. Plehn, and I. Wigmore, Phys. Rev. D 84, 075005 (2011).

[36] A. Ahmadov and M. Demirci, Phys. Rev. D 88, 015017 (2013).

[37] M. Demirci and A. Ahmadov, Phys. Rev. D 89, 075015 (2014).

[38] C. S. Li, Z. Li, R. J. Oakes, and L. L. Yang, Phys. Rev. D 77, 034010 (2008).

[39] J. Debove, B. Fuks, and M. Klasen, Phys. Lett. B 688, 208 (2010).

[40] J. Debove, B. Fuks, and M. Klasen, Nucl. Phys. B842, 51 (2011).
[41] J. Debove, B. Fuks, and M. Klasen, Nucl. Phys. B849, 64 (2011).

[42] B. Fuks, M. Klasen, D. R. Lamprea, and M. Rothering, J. High Energy Phys. 10 (2012) 081.

[43] B. Fuks, M. Klasen, and M. Rothering, J. High Energy Phys. 07 (2016) 053.

[44] L. L. Yang, C. S. Li, J. J. Liu, and Q. Li, Phys. Rev. D 72, 074026 (2005).

[45] A. Broggio, M. Neubert, and L. Vernazza, J. High Energy Phys. 05 (2012) 151.

[46] G. Bozzi, B. Fuks, and M. Klasen, Phys. Rev. D 74, 015001 (2006).

[47] G. Bozzi, B. Fuks, and M. Klasen, Nucl. Phys. B777, 157 (2007).

[48] G. Bozzi, B. Fuks, and M. Klasen, Nucl. Phys. B794, 46 (2008).

[49] B. Fuks, M. Klasen, D. R. Lamprea, and M. Rothering, J. High Energy Phys. 01 (2014) 168.

[50] W. Beenakker, C. Borschensky, M. Kramer, A. Kulesza, E. Laenen, V. Theeuwes, and S. Thewes, J. High Energy Phys. 12 (2014) 023.

[51] C. Borschensky, M. Krmer, A. Kulesza, M. Mangano, S. Padhi, T. Plehn, and X. Portell, Eur. Phys. J. C 74, 3174 (2014).

[52] M. Beneke, J. Piclum, C. Schwinn, and C. Wever, J. High Energy Phys. 10 (2016) 054.

[53] W. Beenakker, C. Borschensky, M. Kramer, A. Kulesza, and E. Laenen, J. High Energy Phys. 12 (2016) 133.

[54] A. Broggio, A. Ferroglia, M. Neubert, L. Vernazza, and L. L. Yang, J. High Energy Phys. 03 (2014) 066.

[55] W. Beenakker, C. Borschensky, R. Heger, M. Kramer, A. Kulesza, and E. Laenen, J. High Energy Phys. 05 (2016) 153.

[56] B. Fuks, M. Klasen, F. Ledroit, Q. Li, and J. Morel, Nucl. Phys. B797, 322 (2008).

[57] T. Jezo, M. Klasen, D. R. Lamprea, F. Lyonnet, and I. Schienbein, J. High Energy Phys. 12 (2014) 092.

[58] M. Mitra, R. Ruiz, D. J. Scott, and M. Spannowsky, Phys. Rev. D 94, 095016 (2016).

[59] M. Klasen, F. Lyonnet, and F. S. Queiroz, Eur. Phys. J. C 77, 348 (2017).

[60] B. Fuks, M. Klasen, D. R. Lamprea, and M. Rothering, Eur. Phys. J. C 73, 2480 (2013).

[61] X. Cid Vidal et al. (Working Group 3 Collaboration), CERN Yellow Rep. Monogr. 7 (2019) 585.

[62] M. Bonvini, S. Marzani, J. Rojo, L. Rottoli, M. Ubiali, R. D. Ball, V. Bertone, S. Carrazza, and N. P. Hartland, J. High Energy Phys. 09 (2015) 191.

[63] W. Beenakker, C. Borschensky, M. Krmer, A. Kulesza, E. Laenen, S. Marzani, and J. Rojo, Eur. Phys. J. C 76, 53 (2016).

[64] J. Fiaschi and M. Klasen, J. High Energy Phys. 03 (2018) 094.

[65] J. Fiaschi, E. Accomando, F. Hautmann, M. Klasen, and S. Moretti, 53rd Rencontres de Moriond on QCD and High Energy Interactions (Moriond QCD 2018): La Thuile, Italy, (ARISF, 2018), ISBN 979-10-96879-06-9.

[66] J. Fiaschi and M. Klasen, Phys. Rev. D 98, 055014 (2018).

[67] J. Fiaschi and M. Klasen, in 2019 European Physical Society Conference on High Energy Physics (EPS-HEP2019), Ghent, Belgium (Proceedings of Science, 2019). 
[68] A. Vogt, Phys. Lett. B 497, 228 (2001).

[69] N. Kidonakis, Int. J. Mod. Phys. A 19, 1793 (2004).

[70] N. Kidonakis, Phys. Rev. D 77, 053008 (2008).

[71] J. Fiaschi, M. Klasen, and M. Sunder, J. High Energy Phys. 04 (2020) 049.

[72] M. Beneke, P. Falgari, and C. Schwinn, Nucl. Phys. B842, 414 (2011).

[73] A. Broggio, A. Ferroglia, M. Neubert, L. Vernazza, and L. L. Yang, J. High Energy Phys. 07 (2013) 042.

[74] S. Dawson, E. Eichten, and C. Quigg, Phys. Rev. D 31, 1581 (1985).

[75] G. Bozzi, B. Fuks, B. Herrmann, and M. Klasen, Nucl. Phys. B787, 1 (2007).

[76] G. F. Sterman, Nucl. Phys. B281, 310 (1987).

[77] S. Catani and L. Trentadue, Nucl. Phys. B327, 323 (1989).

[78] S. Moch, J. A. M. Vermaseren, and A. Vogt, Nucl. Phys. B726, 317 (2005).

[79] O. V. Tarasov, A. A. Vladimirov, and A. Yu. Zharkov, Phys. Lett. 93B, 429 (1980).

[80] S. A. Larin and J. A. M. Vermaseren, Phys. Lett. B 303, 334 (1993).

[81] M. Kramer, E. Laenen, and M. Spira, Nucl. Phys. B511, 523 (1998).

[82] S. Catani, D. de Florian, and M. Grazzini, J. High Energy Phys. 05 (2001) 025.

[83] S. Catani, D. de Florian, M. Grazzini, and P. Nason, J. High Energy Phys. 07 (2003) 028.

[84] M. Beneke, A. Broggio, M. Garny, S. Jaskiewicz, R. Szafron, L. Vernazza, and J. Wang, J. High Energy Phys. 03 (2019) 043.
[85] M. Beneke, A. Broggio, S. Jaskiewicz, and L. Vernazza, J. High Energy Phys. 07 (2020) 078.

[86] M. Beneke, M. Garny, S. Jaskiewicz, R. Szafron, L. Vernazza, and J. Wang, J. High Energy Phys. 01 (2020) 094.

[87] N. Bahjat-Abbas, D. Bonocore, J. Sinninghe Damst, E. Laenen, L. Magnea, L. Vernazza, and C. D. White, J. High Energy Phys. 11 (2019) 002.

[88] T. O. Eynck, E. Laenen, and L. Magnea, J. High Energy Phys. 06 (2003) 057.

[89] C. Duhr, F. Dulat, and B. Mistlberger, arXiv:2001.07717.

[90] A. A. H., G. Das, M. C. Kumar, P. Mukherjee, V. Ravindran, and K. Samanta, arXiv:2001.11377.

[91] G. Aad et al. (ATLAS Collaboration), in XXIX International Symposium on Lepton Photon Interactions at High Energies (LP 2019), Toronto, Canada (Proceedings of Science, 2019), ATLAS-CONF-2019-040.

[92] A. M. Sirunyan et al. (CMS Collaboration), J. High Energy Phys. 10 (2019) 244.

[93] S. Dulat, T.-J. Hou, J. Gao, M. Guzzi, J. Huston, P. Nadolsky, J. Pumplin, C. Schmidt, D. Stump, and C. P. Yuan, Phys. Rev. D 93, 033006 (2016).

[94] W. Porod, Comput. Phys. Commun. 153, 275 (2003).

[95] W. Porod and F. Staub, Comput. Phys. Commun. 183, 2458 (2012).

[96] A. Buckley, J. Ferrando, S. Lloyd, K. Nordstrm, B. Page, M. Rfenacht, M. Schnherr, and G. Watt, Eur. Phys. J. C 75, 132 (2015).

[97] M. Tanabashi et al. (Particle Data Group), Phys. Rev. D 98, 030001 (2018).

[98] M. Aaboud et al. (ATLAS Collaboration), Eur. Phys. J. C 78, 154 (2018). 